\title{
The Hyper-MUCHFUSS project: probing the Galactic halo with sdB stars ${ }^{\star}$
}

\author{
A. Tillich ${ }^{1}$, U. Heber ${ }^{1}$, S. Geier ${ }^{1}$, H. Hirsch ${ }^{1}$, P. F. L. Maxted ${ }^{2}$, B. T. Gänsicke ${ }^{3}$, T. R. Marsh ${ }^{3}$, R. Napiwotzki ${ }^{4}$, \\ R. H. Østensen ${ }^{5}$, and R.-D. Scholz ${ }^{6}$ \\ 1 Dr. Karl Remeis-Observatory \& ECAP, Astronomical Institute, Friedrich-Alexander University Erlangen-Nuremberg, \\ Sternwartstr. 7, 96049 Bamberg, Germany \\ e-mail: Alfred.Tillich@sternwarte.uni-erlangen.de \\ 2 Astrophysics Group, School of Physics and Geographical Sciences, Lennard-Jones Laboratories, Keele University, ST5 5BG, UK \\ Department of Physics, University of Warwick, Coventry CV4 7AL, UK \\ ${ }^{4}$ Centre of Astrophysics Research, University of Hertfordshire, College Lane, Hatfield AL10 9AB, UK \\ Instituut voor Sterrenkunde, K.U. Leuven, Celestijnenlaan 200D, 3001 Leuven, Belgium \\ ${ }^{6}$ Astrophysikalisches Institut Potsdam, An der Sternwarte 16, 14482 Potsdam, Germany
}

Received 6 August 2010 / Accepted 22 November 2010

\begin{abstract}
Context. High-velocity stars in the Galactic halo, e.g. the so-called hyper-velocity stars (HVS), are important tracers of the properties of the dark matter halo, in particular its mass.

Aims. A search for the fastest stars among hot subdwarfs (sdB) in the halo is carried out to identify HVS, unbound to the Galaxy, and bound population II stars, in order to derive a lower limit to the halo mass.

Methods. Based on the SDSS DR6 spectral database we selected stars with high rest-frame velocities. These radial velocity (RV) measurements were verified at several telescopes to exclude RV variable stars. Out of 88 stars observed in the follow-up campaign, 39 stars were found to have constant RVs. For twelve of them we measured a proper motion significantly different from zero and obtained spectroscopic distances from quantitative spectral analysis to construct the full $6 \mathrm{D}$ phase space information for a kinematical study.

Results. All but one programme sdB show halo characteristics, but can be distinguished as two kinematical groups, one (G1) with the low Galactic rotation typical of halo stars and a second one $(\mathrm{G} 2)$ with rapid retrograde motion. We also investigate the possibility that the programme stars are not genuine halo stars but ejected from the Galactic disc or bulge. The G1 objects crossed the Galactic plane in the central bulge, whereas the G2 stars did so in the outer Galactic disc. J1211+1437 (G2) is an HVS candidate, as it is unbound to the Galaxy if the standard Galactic potential is adopted.

Conclusions. We conclude that in the ejection scenario, G1 stars might have been formed via the slingshot mechanism that invokes acceleration by tidal interaction of a binary with the central supermassive black hole. The G2 stars, however, would originate in the outskirts of the Galactic disc and not in the central bulge. J1211+1437 is the first unbound subdwarf B star, for which we can rule out the slingshot mechanism. Alternatively, we may assume that the stars are old population II stars and so they have to be bound. Then the kinematics of $\mathrm{J} 1211+1437$ set a lower limit of $2 \times 10^{12} M_{\odot}$ to the mass of the Galactic dark matter halo.
\end{abstract}

Key words. stars: kinematics and dynamics - subdwarfs - line: profiles - proper motions - Galaxy: halo - stars: atmospheres

\section{Introduction}

The properties of the dark matter halo are important for understanding how the Galaxy formed and evolved. Observations of halo stars put constraints on theoretical models of halo formation (e.g. Navarro et al. 1996). Large surveys, such as the Sloan Digital Sky Survey (SDSS, York et al. 2000) and the RAdial Velocity Experiment (RAVE, Steinmetz et al. 2006), provide large numbers of stars for tracing the halo properties, such as the total mass of the halo.

Globular clusters, satellite galaxies, as well as large samples of halo stars have been used to estimate the halo mass. Actually only the objects with the most extreme velocities provide tight

* Based on data from the Sloan Digital Sky Survey, and data collected at the $3.5 \mathrm{~m}$ telescope at DSAZ observatory (Calar Alto) in Spain, the $4.2 \mathrm{~m}$ William Herschel Telescope on La Palma, and the European Southern Observatory in Chile according 081.D-0819(A), 082.D-0649(A) and 084.D-0348(A). constraints, so that the mass estimates depend mostly on them (Sakamoto et al. 2003; Smith et al. 2007). A halo mass of about $2 \times 10^{12} M_{\odot}$ was favoured in earlier investigations (Wilkinson \& Evans 1999; Sakamoto et al. 2003), while more recent studies prefer lower masses of about half that value (Battaglia et al. 2005; Smith et al. 2007; Xue et al. 2008).

The hyper-velocity stars (HVS, Brown et al. 2005; Hirsch et al. 2005; Edelmann et al. 2005) are the fastest moving stars known in the halo. Their supposed place of origin is the Galactic centre (GC), where it has been suggested that they are accelerated by tidal interactions of a binary star with the super-massive black hole (SMBH, Hills 1988). Whether an HVS can in fact escape from the Galaxy or not depends on the halo mass (Abadi et al. 2009).

Kinematical studies of the hyper-velocity stars were based on their radial velocities (RVs) alone. Recently, Tillich et al. (2009) were able to measure proper motions of an A-type HVS and study its 3-D kinematics to trace its place of birth in the 
Galactic disc. They found it to originate far from the GC, thereby challenging the SMBH-slingshot mechanism of Hills (1988). Tillich et al. (2009) thus suggested a runaway mechanism for the star's formation. Further evidence that such a mechanism works comes from two similar studies of the hyper runaway stars HD 217791 (Heber et al. 2008) and HIP 60350 (Irrgang et al. 2010), which were also found to originate in the outer rim of the Galactic disc nowhere near the GC.

While most of the 17 HVS known today (Brown et al. 2009; Tillich et al. 2009) are early-type main-sequence stars, there is just one evolved low-mass star, US 708, a hot subdwarf star of spectral type sdO (Hirsch et al. 2005).

Most of the previous studies of halo stars to constrain the dark matter properties are hampered by the lack of proper motion measurements. Hence they had to rely substantially on RV distributions. In such cases only four coordinates (i.e. two position values, distance and RV) of the $6 \mathrm{D}$ phase space are determined and the missing proper motion components are handled in a statistical approach. In the presently most extensive study Xue et al. (2008) measured RVs for more than 10000 blue halo stars from the SDSS and classified their sample as a mix of blue horizontal branch (BHB) stars, blue stragglers and main-sequence stars with effective temperatures roughly between 7000 and $10000 \mathrm{~K}$ according to their colours. Xue et al. (2008) selected 2400 BHB stars to estimate the halo mass out to $60 \mathrm{kpc}$ to be $1.0 \times 10^{12} M_{\odot}$ using a halo model of Navarro et al. (1997). For one star from that sample Przybilla et al. (2010) were able to obtain proper motion and carry out a detailed kinematic analysis, which revealed an inbound Population II horizontal branch star with a Galactic rest-frame (GRF) velocity of $\sim 700 \mathrm{~km} \mathrm{~s}^{-1}$ at its current position. This makes it the fastest halo star known, and provided a lower limit of $1.7 \times 10^{12} M_{\odot}$ for the total halo mass of the Galaxy, significantly exceeding the value determined by Xue et al. (2008).

This example shows that it is rewarding to study the kinematics of additional stars in the halo and to consider classes of stars other than BHB stars, as well. Of course, the Galactic halo hosts a plethora of white dwarfs (Eisenstein et al. 2006). However, they are so faint that they can only be analysed in the solar neighbourhood. Another group of evolved low-mass stars are the hot subdwarf stars $(\mathrm{sdB}, \mathrm{sdO})$ that dominate the population of faint blue stars at high Galactic latitudes to visual magnitudes of about $V=18$ (Green et al. 1986). They are considered to be helium-core burning stars with very thin $\left(<0.02 M_{\odot}\right)$, inert hydrogen envelopes and masses around $0.5 M_{\odot}$. Following ideas outlined by Heber (1986), the sdBs can be identified with models for extreme horizontal branch (EHB) stars. An EHB star bears a strong resemblance to a helium main-sequence star of half a solar mass, and it should evolve similarly, i.e. directly to the white dwarf cooling sequence, bypassing a second giant phase (for a review see Heber 2009). For the formation of subdwarf B stars, three scenarios are discussed by Han et al. (2003): common envelope ejection, stable Roche lobe overflow (RLOF), and the merger of two helium white dwarfs. Some alternate scenarios for the formation of single sdB stars are reviewed by $\emptyset$ stensen (2009).

Hot subdwarf stars exist not only in the field of the Galaxy but also in globular clusters, in the Galactic bulge and have even been resolved in the elliptical galaxy M 32 (Brown et al. 2008). Kinematical studies (Altmann et al. 2004; Napiwotzki 2008) indicate that they occur in all stellar populations of the Galaxy.

However, very little is known about the halo population of hot subdwarfs except those in globular clusters. Some highvelocity hot subdwarfs have attracted interest because of their high RVs, most notably, the sdO star US 708, whose RV in the rest-frame was measured at $751 \mathrm{~km} \mathrm{~s}^{-1}$ (Hirsch et al. 2005) the second HVS star discovered. Unfortunately, we cannot deduce the origin of the star, as we lack a reliable proper motion measurement.

Motivated by the discovery of US 708, we embarked on a project to identify a sample of population II hot subdwarfs and study their kinematics from RV and proper motion. We make use of the MUCHFUSS survey (Geier et al. 2011a), which searches for close binaries with high RV variations. The search strategy also provides targets that are not close binaries but instead travel through space at high RV without variations. These stars are the targets of our investigation. Accordingly, we entitled our project Hyper-MUCHFUSS as we provide an extension of MUCHFUSS.

The paper is organised as follows. In Sect. 2 we introduce our survey for HVSs and in Sect. 3 our sophisticated proper motion measurement method. The kinematical analysis techniques are shown in Sect. 4. In Sect. 5 we present our results and summarise and conclude in Sect. 6.

\section{Survey}

The enormous SDSS database is evaluated well in terms of errors and accuracy. This makes it the perfect starting point for the MUCHFUSS survey. To select subdwarf candidates, we used several indicators, e.g. colour, spectral classification, and RV. Figure 1 shows a flowchart of the target selection method. We selected sdO/B candidates by colour $(g-r<0.1$ and $u-g<0.4$, see Geier et al. 2011a) and pre-classified their spectra by visual inspection. Measuring the RV by fitting synthetic models, we selected only stars faster than $\pm 100 \mathrm{~km} \mathrm{~s}^{-1}$. For most of the known sdB binaries, the RV semi-amplitudes are below $100 \mathrm{~km} \mathrm{~s}^{-1}$ (Geier et al. 2011a), so that the RV of a typical sdB binary of the Galactic disc will rarely exceed $100 \mathrm{~km} \mathrm{~s}^{-1}$ in absolute value, and these stars are consequently excluded in MUCHFUSS. We converted the heliocentric RV to the Galactic rest-frame (GRF). The higher the RV, the higher the priority we assign to the target for our survey. Especially stars with absolute GRF velocities of more than $275 \mathrm{~km} \mathrm{~s}^{-1}$ are high priority HVS candidates. This observational cut was introduced by Brown et al. (2007) to distinguish HVS from halo stars by their origin. More information on MUCHFUSS and the process of target selection is presented by Geier et al. (2011a).

More than 250 targets with measured RV remained on the MUCHFUSS target list, serving as a first epoch. Second-epoch spectra were obtained with ESO-VLT/FORS $(R \approx 1800, \lambda \approx$ $3730-5200 \AA)$, WHT/ISIS $(R \approx 1800, \lambda \approx 3730-5200 \AA)$, CAHA-3.5 m/TWIN $(R \approx 4000, \lambda \approx 3460-5630 \AA)$ and ESONTT/EFOSC $2(R \approx 2200, \lambda \approx 4450-5110 \AA)$. Due to the different wavelength coverage and resolution, the number of visible absorption lines is correspondingly restricted ${ }^{1}$. Up to now, such spectra are available for 88 stars, which is about one third of the target list. The RVs have been measured by $\chi^{2}$-fitting of suitable synthetic spectra. Based on the MUCHFUSS RV list, we regard a star as RV constant if its velocity is consistent with the first epoch within the respective error limits; however, it is obvious that these errors individually depend on a variety of conditions, such as the $\mathrm{S} / \mathrm{N}$ (see Table 1), the resolution, wavelength coverage, and the number of visible absorption lines.

The MUCHFUSS results for the binaries will be presented in a dedicated paper by Geier et al. (2011b). While 49 of the target stars were found to be RV variable, 39 do not show any

1 The EFOSC2 spectra only cover $\mathrm{H}_{\beta}$ and $2 \mathrm{He}$ I lines. 
A. Tillich et al.: The Hyper-MUCHFUSS project: probing the Galactic halo with sdB stars

Table 1. Heliocentric RV table of the target sample with $\mathrm{S} / \mathrm{N}$ per pixel in the continuum next to $\mathrm{H}_{\beta}$.

\begin{tabular}{|c|c|c|c|c|c|c|}
\hline Name & Short name & Type & OBS & MJD & $\begin{array}{l}v_{\mathrm{rad}} \\
\mathrm{km} \mathrm{s}^{-1}\end{array}$ & $\mathrm{SNR} @ \mathrm{H}_{\beta}$ \\
\hline \multirow[t]{2}{*}{ SDSSJ084447.00+113910.0 } & J0844+1139 & $\mathrm{sdB}$ & SDSS & 2453801.1251331 & $+202.7 \pm 5.0$ & 52 \\
\hline & & & NTT & 2454755.86312183 & $+219.5 \pm 30.4$ & 13 \\
\hline \multirow{2}{*}{ SDSSJ084556.85+135211.3 } & $\mathrm{J} 0845+1352$ & $\mathrm{sdB}$ & SDSS & 2453799.765353 & $+115.1 \pm 9.6$ & 33 \\
\hline & & & NTT & 2454756.86725607 & $+92.0 \pm 15.1$ & 22 \\
\hline \multirow[t]{3}{*}{ SDSSJ094850.47+551631.7 } & $\mathrm{J} 0948+5516$ & $\mathrm{sdB}$ & SDSS & 2451991.6335069 & $-138.3 \pm 12.2$ & 17 \\
\hline & & & WHT & 2454587.46586414 & $-158.5 \pm 17.8$ & 9 \\
\hline & & & WHT & 2454588.38885923 & $-127.4 \pm 6.6$ & 15 \\
\hline \multirow[t]{2}{*}{ SDSSJ102057.16+013751.3 } & $\mathrm{J} 1020+0137$ & $\mathrm{sdB}$ & SDSS & 2451997.186412 & $+243.3 \pm 8.3$ & 48 \\
\hline & & & FORS & 2454638.47970756 & $+239.4 \pm 7.8$ & 36 \\
\hline \multirow{2}{*}{ SDSSJ121150.27+143716.2 } & $\mathrm{J} 1211+1437$ & $\mathrm{sdB}$ & SDSS & 2453466.7114641 & $+220.2 \pm 9.5$ & 32 \\
\hline & & & FORS & 2454575.76860445 & $+207.5 \pm 25.6$ & 11 \\
\hline \multirow[t]{2}{*}{ SDSSJ155635.80+470851.8 } & $\mathrm{J} 1556+4708$ & $\mathrm{sdB}$ & SDSS & 2452354.493235 & $-393.5 \pm 19.9$ & 18 \\
\hline & & & WHT & 2454586.60330613 & $-385.4 \pm 7.4$ & 14 \\
\hline \multirow[t]{2}{*}{ SDSSJ163213.05+205124.0 } & $\mathrm{J} 1632+2051$ & $\mathrm{sdB}$ & SDSS & 2453224.6422685 & $-238.6 \pm 6.7$ & 38 \\
\hline & & & FORS & 2454656.62186841 & $-237.9 \pm 2.8$ & 45 \\
\hline \multirow[t]{2}{*}{ SDSSJ164419.44+452326.7 } & $\mathrm{J} 1644+4523$ & $\mathrm{sdB}$ & SDSS & 2452051.9386574 & $-314.1 \pm 4.5$ & 32 \\
\hline & & & WHT & 2454586.71567501 & $-312.8 \pm 11.6$ & 31 \\
\hline \multirow[t]{2}{*}{ SDSSJ215648.71+003620.7 } & $\mathrm{J} 2156+0036$ & $\mathrm{sdB}$ & SDSS & 2452933.5838194 & $-177.2 \pm 6.5$ & 32 \\
\hline & & & NTT & 2454757.70342858 & $-190.1 \pm 37.2$ & 13 \\
\hline \multirow[t]{2}{*}{ SDSSJ224451.81+010630.9 } & $\mathrm{J} 2244+0106$ & $\mathrm{sdB}$ & SDSS & 2452146.8122801 & $-166.0 \pm 5.9$ & 24 \\
\hline & & & NTT & 2454756.61349441 & $-149.1 \pm 19.2$ & 17 \\
\hline \multirow[t]{2}{*}{ SDSSJ084938.85+145503.2 } & $\mathrm{J} 0849+1455$ & DA & SDSS & 2453818.6548958 & $+119.0 \pm 37.2$ & 15 \\
\hline & & & NTT & 2454757.87125523 & $+82.6 \pm 33.9$ & 9 \\
\hline \multirow[t]{2}{*}{ SDSSJ135824.61+472931.6 } & $\mathrm{J} 1358+4729$ & DA & SDSS & 2452723.9695428 & $-260.6 \pm 14.2$ & 24 \\
\hline & & & WHT & 2454586.54874204 & $-257.0 \pm 14.3$ & 22 \\
\hline
\end{tabular}
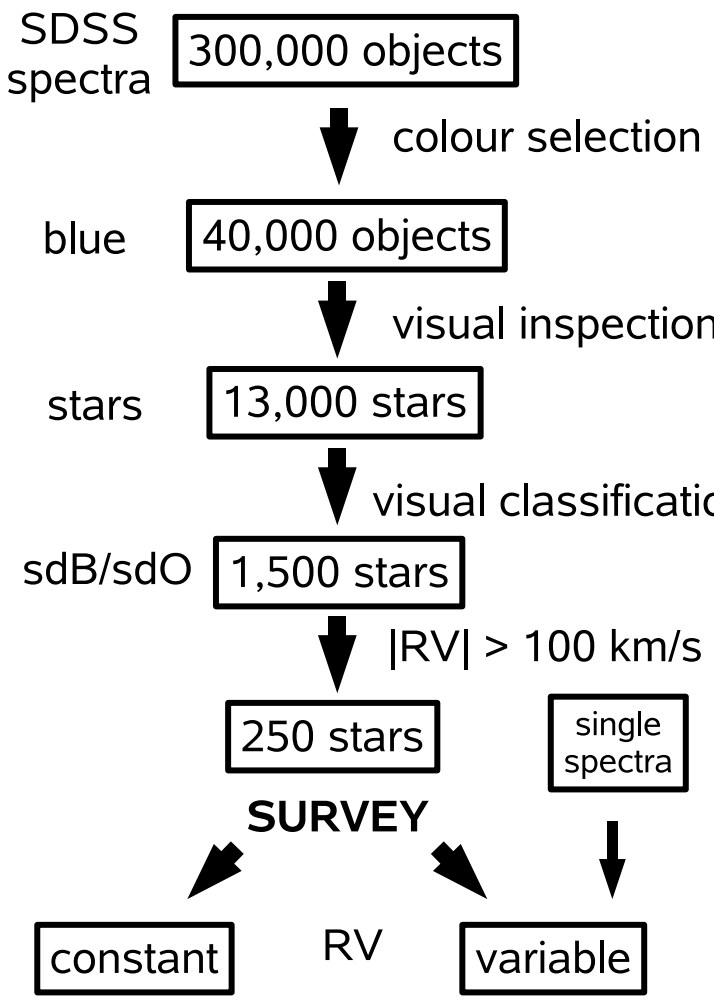

Fig. 1. Target selection method based on SDSS. Stars are selected for which the absolute value of the RV exceeds $100 \mathrm{~km} \mathrm{~s}^{-1}$. Hence disc stars are efficiently removed.

variations and therefore make up the sample that is studied in this paper. In the following sections we focus exclusively on these candidates.

\section{Proper motion}

A kinematical analysis can only be done if the star's location and space velocity is known, so we attempt to measure proper motions and determine spectroscopic distances for all our 39 candidates. We collected all available independent position measurements on Schmidt plates (APM - McMahon et al. 2000; SSS - Hambly et al. 2001) and combined them with the SDSS and other available positions (CMC14 Carlsberg-Meridian-Catalog 2006; 2MASS - Cutri et al. 2003; UKIDSS - Lawrence et al. 2007). Multi-epoch SDSS astrometric measurements taken from the Princeton SDSS database (Finkbeiner et al. 2004) were included in the proper motion fit. We obtained more measurements of Schmidt plates from up to 14 different epochs in case of overlapping plates of the Digitised Sky Survey ${ }^{2}$ (DSS). The FITS images of 15 by 15 arcmin size were extracted from all available plates and the ESO MIDAS tool Centre/Gauss was used to measure positions. To measure absolute proper motions, we initially had to find and identify compact background galaxies from the SDSS. It is very important to obtain enough galaxies that are distributed well over the image, although galaxies tend to cluster. We used at least 10 galaxies per measurement and determined the position of the star relative to the reference galaxies. Finally we transformed the target positions on all the Schmidt plates to the SDSS system. The small fields allowed us to apply a simple model (shift+rotation). The typical error in both coordinates at one epoch, including the error of the transformation using the measured galaxies and the individual centroiding error for the target, was 150 mas. We obtained one position per epoch and used linear regression to derive the proper motions with their errors (see e.g. Fig. 2).

\footnotetext{
${ }^{2}$ http://archive.stsci.edu/cgi-bin/dss_plate_finder
} 

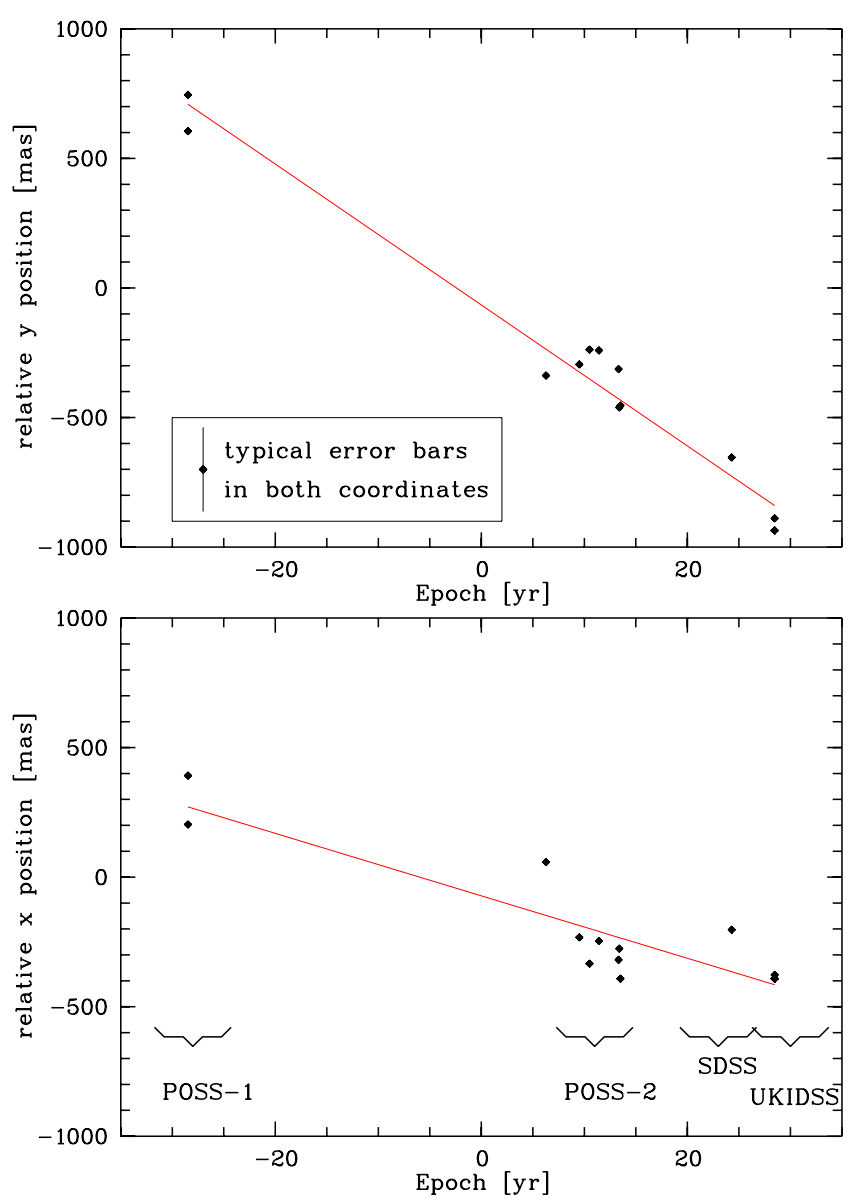

Fig. 2. Proper motion components derived from the position measurements for $\mathrm{J} 1211+1437$, where 1978.77 is the zero epoch.

Especially for the old POSS-I epoch, we noticed large discrepancies between the positions, which are probably due to the different colour filters and quality of the plates. The best way to minimise possible systematic effects is simply to use the same set of reference galaxies for every epoch. We regard a proper motion as detected, if the position measurements do not show a wide spread relative to the linear fit, and the derived proper motion is significantly different from zero. 27 stars turned out to have a proper motion consistent with zero, while a significant proper motion was measured for 12 of our 39 candidates $(\approx 38 \%)$. One of them is the sdB J2156+0036, which has already been used to show the potential of our method (see Fig. 1 of Tillich et al. 2010a). This star also represents a typical star with proper motion from our target sample. For all but two objects (J1644+4523 and J0849+1455), the determined proper motions agree within their errors with those of the recently published PPMXL catalogue (Roeser et al. 2010), where our proper motion errors are smaller than the PPMXL ones for all objects.

Tables 1 and 2 list the measurements for the sample analysed in this paper. In Fig. 2 we demonstrate the measurement of the proper motion for $\mathrm{J} 1211+1437$, an outstanding target that will be discussed in detail in Sect. 5.1.

\section{Distance and kinematics}

The second important parameter is the distance, needed to determine the current location of the stars and their transversal velocities, along with their proper motions. We determine the distance
Table 2. PM measurements for the positive detections together with the number of epochs $n_{\mathrm{ep}}$ and the number of reference galaxies $n_{\mathrm{gal}}$.

\begin{tabular}{lllll}
\hline \hline Name & $\begin{array}{l}V \\
\text { mag }\end{array}$ & $\begin{array}{l}\left(\mu_{\alpha} \cos (\delta)\right) \\
\operatorname{mas} / \mathrm{yr}\end{array}$ & $\begin{array}{l}\mu_{\delta} \\
\mathrm{mas} / \mathrm{yr}\end{array}$ & $n_{\mathrm{ep}} / n_{\mathrm{gal}}$ \\
\hline $\mathrm{J} 0844+1139$ & $16.11 \pm 0.02$ & $-11.5 \pm 2.6$ & $-11.9 \pm 2.1$ & $6 / 7$ \\
$\mathrm{~J} 0845+1352$ & $17.42 \pm 0.02$ & $-10.7 \pm 2.3$ & $-3.7 \pm 2.6$ & $5 / 14$ \\
$\mathrm{~J} 0948+5516$ & $18.59 \pm 0.03$ & $-1.9 \pm 2.9$ & $-7.4 \pm 1.7$ & $7 / 12$ \\
$\mathrm{~J} 1020+0137$ & $16.91 \pm 0.02$ & $-2.4 \pm 1.3$ & $-7.3 \pm 1.2$ & $12 / 15$ \\
$\mathrm{~J} 1211+1437$ & $17.87 \pm 0.03$ & $-12.1 \pm 1.8$ & $-27.2 \pm 1.4$ & $13 / 14$ \\
$\mathrm{~J} 1556+4708$ & $18.61 \pm 0.03$ & $-6.3 \pm 1.9$ & $+1.3 \pm 1.8$ & $10 / 14$ \\
$\mathrm{~J} 1632+2051$ & $17.62 \pm 0.03$ & $-12.5 \pm 3.0$ & $-1.6 \pm 3.6$ & $7 / 16$ \\
$\mathrm{~J} 1644+4523$ & $17.36 \pm 0.02$ & $+4.7 \pm 2.8$ & $-26.1 \pm 3.3$ & $8 / 13$ \\
$\mathrm{~J} 2156+0036$ & $17.91 \pm 0.03$ & $-1.3 \pm 1.6$ & $-7.4 \pm 1.2$ & $26 / 13$ \\
$\mathrm{~J} 2244+0106$ & $18.14 \pm 0.02$ & $+2.1 \pm 0.8$ & $-3.7 \pm 1.3$ & $30 / 14$ \\
$\mathrm{~J} 0849+1455$ & $18.85 \pm 0.03$ & $-49.5 \pm 1.8$ & $+6.4 \pm 1.6$ & $8 / 9$ \\
$\mathrm{~J} 1358+4729$ & $18.03 \pm 0.03$ & $+76.1 \pm 2.4$ & $+14.4 \pm 2.4$ & $14 / 15$ \\
\hline
\end{tabular}

Notes. The $V$ magnitudes have been derived from SDSS photometry using the transformations of Jordi et al. (2006).

from a quantitative spectral analysis, which provides the effective temperature, gravity, and helium abundance. The distance is derived from the atmospheric parameters and the apparent magnitude, by adopting the canonical mass of $0.48 M_{\odot}$.

In most of the cases, the stellar spectra from the SDSS provide sufficient $\mathrm{S} / \mathrm{N}$ and resolution for a quantitative spectral analysis. Furthermore, in some cases they make up the most reliable data we have. We applied $\chi^{2}$-fitting of synthetic line profiles to the Balmer and helium lines in order to determine the atmospheric parameters and abundances (see Fig. 6). Inevitably, our selection procedure produces some misclassifications, therefore we also ended up with two DA white dwarfs in our sample. All of the subdwarfs have been analysed using fully metal, lineblanketed LTE models of solar metallicity (Heber et al. 2000) ${ }^{3}$. Finally spectroscopic distances were calculated using the astrophysical fluxes following Ramspeck et al. (2001). Independent studies showed that the atmospheric parameters (hence the distances) only depend a little on the choice of the metallicity. Heber et al. (2000) studied the effect by comparing results from models with solar composition to those of $1 / 100$ solar metallicity in a detailed study of high-resolution spectra for three sdB stars. The differences range from 200 to $300 \mathrm{~K}$ in $T_{\text {eff }}$ and 0.03 dex in $\log g$, far lower than the uncertainties of our results, so the metallicity effects are not significant. The uncertainties of the distance have been determined using a Monte Carlo approach for the input quantities (cf. Tillich et al. 2009, 2010b; Przybilla et al. 2010).

By applying the Galactic potential of Allen \& Santillan (1991) we calculated orbits and reconstructed the path of the star with the program of Odenkirchen \& Brosche (1992). The distance of the GC from the Sun was adopted to be $8.0 \mathrm{kpc}$ and the Sun's motion with respect to the local standard of rest was taken from Dehnen \& Binney (1998). Based on our data, we are not able to draw conclusions on detailed structural properties of different galactic potentials. However, we are able to discuss global effects (see Sect. 5). The error in space motion is dominated by that of the distance (via the gravity error) and those of the proper motion components ${ }^{4}$. When varying these

\footnotetext{
3 For the DA white dwarfs we used the synthetic models by Koester (2009).

4 Although some of the second-epoch spectra are of low quality, the RV errors (see Table 1) are irrelevant for the error budget. The distribu-
} 


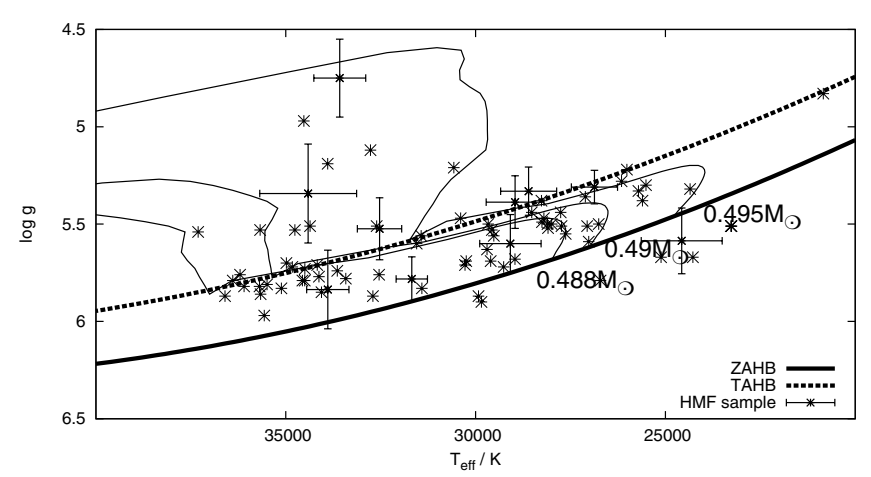

Fig. 3. Comparison of the position of the 10 subdwarfs (with error bars) from the HMF project in the $T_{\text {eff }}-\log g$-diagram to evolutionary tracks of Dorman et al. (1993) for a metallicity of $[\mathrm{Fe} / \mathrm{H}]=-1.48$. The ZAHB and the TAHB are only indicated from fitting of the tracks. The SPY subdwarf B sample is plotted for reference (Lisker et al. 2005). Two subdwarfs lie lie well above the canonical TAHB and therefore are most likely in a shell-He burning post-EHB stage of evolution.

three quantities within their respective errors we applied a Monte Carlo procedure for the error propagation and for deriving the median GRF velocities at the present location and their distribution (see Fig. 8, cf. Tillich et al. 2009, 2010b; Przybilla et al. 2010). We compared our results with the local escape velocities as calculated from the Galactic potential of Allen \& Santillan (1991). Furthermore, from kinematic characteristics $(U, V$, eccentricity $e$, and the $z$-component of the angular momentum $J_{Z}$ ), we obtained additional information about the population membership of the stars. By following the trajectories backwards in time, we can even put constraints on the origin of the stars, when applying the same Monte Carlo method.

\section{Results}

Until now about one third of the targets of the MUCHFUSS survey have been observed. Accordingly, 39 of them have constant RV within the detection limits. Twelve stars showed proper motions significantly different from zero and were analysed in detail using quantitative spectroscopy. In Fig. 6 we show a comparison between the observed and the synthetic spectra for two subdwarf stars. Two stars turned out to be DA white dwarfs rather than sdB stars. The derived stellar parameters are shown in Table 3, while the respective RV can be found in Table 4.

In Fig. 3 we show a $T_{\text {eff- }} \log g$-diagram for the 10 subdwarfs in comparison to the reference sample of the SPY sdB stars analysed by Lisker et al. (2005). Obviously eight of our subdwarf B stars reside on the EHB within the respective errors. However, the stars J1556+4708 and J2244+0106 lie well above the EHB, and must therefore be in a post-EHB stage of evolution if they have the canonical mass of $0.48 M_{\odot}$. Figure 3 shows that our subdwarf B sample goes well with the SPY sample. Based on these parameters we determined the distances, which are given in Table 4. Together with the median space velocity we also obtained the escape velocity in the Galactic potential of Allen \& Santillan (1991). To further quantify the kinematics of the stars, we made a comparison with the kinematics of white dwarfs. A sample of 398 white dwarfs from the SPY survey was studied by Pauli et al. (2006). They introduced a kinematic population

tion of the space velocity is dominated by the errors in the transversal velocity components, which may have uncertainties as large as $\pm 100 \mathrm{~km} \mathrm{~s}^{-1}$.
Table 3. Stellar parameters for the remaining stars with PM significantly different from zero.

\begin{tabular}{lcccc}
\hline \hline Name & Type & $\begin{array}{c}T_{\text {eff }} \\
\mathrm{K}\end{array}$ & $\log g$ & $\log n_{\mathrm{He}} / n_{\mathrm{H}}$ \\
& & & \\
\hline $\mathrm{J} 0844+1139$ & $\mathrm{sdB}$ & $28600 \pm 740$ & $5.33 \pm 0.12$ & $-2.6 \pm 0.4$ \\
$\mathrm{~J} 0845+1352$ & $\mathrm{sdB}$ & $24570 \pm 1070$ & $5.59 \pm 0.17$ & $<-3.0$ \\
$\mathrm{~J} 0948+5516$ & $\mathrm{sdB}$ & $33890 \pm 560$ & $5.84 \pm 0.20$ & $-1.5 \pm 0.2$ \\
$\mathrm{~J} 1020+0137$ & $\mathrm{sdB}$ & $28960 \pm 760$ & $5.39 \pm 0.14$ & $<-3.0$ \\
$\mathrm{~J} 1211+1437$ & $\mathrm{sdB}$ & $32530 \pm 580$ & $5.52 \pm 0.16$ & $-2.9 \pm 0.5$ \\
$\mathrm{~J} 1556+4708$ & $\mathrm{sdB}$ & $34410 \pm 1280$ & $5.34 \pm 0.25$ & $<-3.0$ \\
$\mathrm{~J} 1632+2051$ & $\mathrm{sdB}$ & $26870 \pm 610$ & $5.31 \pm 0.09$ & $-2.1 \pm 0.2$ \\
$\mathrm{~J} 1644+4523$ & $\mathrm{sdB}$ & $31680 \pm 410$ & $5.78 \pm 0.11$ & $-2.9 \pm 0.3$ \\
$\mathrm{~J} 2156+0036$ & $\mathrm{sdB}$ & $29080 \pm 810$ & $5.60 \pm 0.15$ & $-2.5 \pm 0.3$ \\
$\mathrm{~J} 2244+0106$ & $\mathrm{sdB}$ & $33580 \pm 680$ & $4.75 \pm 0.20$ & $-1.5 \pm 0.2$ \\
$\mathrm{~J} 0849+1455$ & $\mathrm{DA}$ & $27750 \pm 830$ & $7.32 \pm 0.15$ & - \\
$\mathrm{J} 1358+4729$ & $\mathrm{DA}$ & $10640 \pm 110$ & $8.10 \pm 0.07$ & - \\
\hline
\end{tabular}

Table 4. Estimated distance, escape and GRF velocities (median) and local escape velocities for the Galactic potential of Allen \& Santillan (1991).

\begin{tabular}{lcccr}
\hline \hline Name & $\begin{array}{c}\text { Dist } \\
\mathrm{kpc}\end{array}$ & $\begin{array}{c}v_{\mathrm{rad}} \\
\mathrm{km} \mathrm{s}^{-1}\end{array}$ & $\begin{array}{c}v_{\mathrm{GRF}} \\
\mathrm{km} \mathrm{s}^{-1}\end{array}$ & $\begin{array}{c}v_{\mathrm{esc}} \\
\mathrm{km} \mathrm{s}^{-1}\end{array}$ \\
\hline $\mathrm{J} 0844+1139$ & $3.14 \pm 0.26$ & $202.7 \pm 5.0$ & $202_{-46}^{+55}$ & 513 \\
$\mathrm{~J} 0845+1352$ & $3.50 \pm 0.38$ & $115.1 \pm 9.6$ & $230_{-61}^{+70}$ & 511 \\
$\mathrm{~J} 0948+5516$ & $6.51 \pm 0.88$ & $-142.1 \pm 8.6$ & $106_{-35}^{+78}$ & 490 \\
$\mathrm{~J} 1020+0137$ & $4.31 \pm 0.39$ & $238.0 \pm 8.3$ & $107_{-13}^{+20}$ & 513 \\
$\mathrm{~J} 1211+1437$ & $6.45 \pm 0.68$ & $220.1 \pm 9.5$ & $713_{-139}^{+155}$ & 507 \\
$\mathrm{~J} 1556+4708$ & $11.76 \pm 2.03$ & $-385.4 \pm 7.4$ & $353_{-106}^{+174}$ & 487 \\
$\mathrm{~J} 1632+2051$ & $6.09 \pm 0.36$ & $-236.1 \pm 3.2$ & $275_{-110}^{+120}$ & 544 \\
$\mathrm{~J} 1644+4523$ & $3.68 \pm 0.27$ & $-314.1 \pm 4.5$ & $468_{-90}^{+104}$ & 534 \\
$\mathrm{~J} 2156+0036$ & $5.37 \pm 0.54$ & $-177.2 \pm 6.5$ & $85_{-44}^{+58}$ & 534 \\
$\mathrm{~J} 2244+0106$ & $18.29 \pm 2.45$ & $-166.0 \pm 5.8$ & $200_{-156}^{+186}$ & 458 \\
$\mathrm{~J} 0849+1455$ & $1.11 \pm 0.11$ & $119.0 \pm 37.2$ & $354_{-34}^{+33}$ & 531 \\
$\mathrm{~J} 1358+4729$ & $0.14 \pm 0.01$ & $-260.6 \pm 14.2$ & $295_{-11}^{+10}$ & 541 \\
\hline
\end{tabular}

classification scheme based on the 3D-orbit, the $V-U$ diagram, and the $e$ - $J_{\mathrm{Z}}$-diagram and combined it with age information. They performed a detailed kinematical analysis accounting for errors by means of a Monte Carlo error propagation code, similar to our method. A sample of abundance selected typical mainsequence stars served as reference sample. They derived the $3 \sigma-$ contours for the $V-U$ diagram on which the kinematic classification is based. For the $e-J_{Z}$ diagram a "Region B" is defined such that it excludes as many thick-disc stars as possible. The last criterion is the $\rho$-Z-diagram, which is used to classify the orbits among the populations by comparing with template Galactic orbits (Pauli et al. 2003). A substantial thick-disc fraction of $7 \%$ was found, while only $2 \%$ of the DAs show characteristic halo properties.

In Fig. 4 we compare our sdB sample to the white dwarf sample of Pauli et al. (2003). All sdB stars lie far away from the thin-disc population of the white dwarfs. They are found in those regions of both diagrams where very few white dwarfs lie; only the rare ones belonging to the halo population. The sdB J0845+1352 shows thick-disc kinematics (see Fig. 4). Nine sdB stars possess halo characteristics, since they clearly reside outside the $3 \sigma$ thick-disc contour in the diagrams. According to the $e-J_{\mathrm{Z}}$ diagram, the sdBs can be divided into two subgroups. Furthermore, the 3D-orbits (see Fig. 5 for examples of an G1 and a G2 star, respectively) all clearly favour a halo membership. 

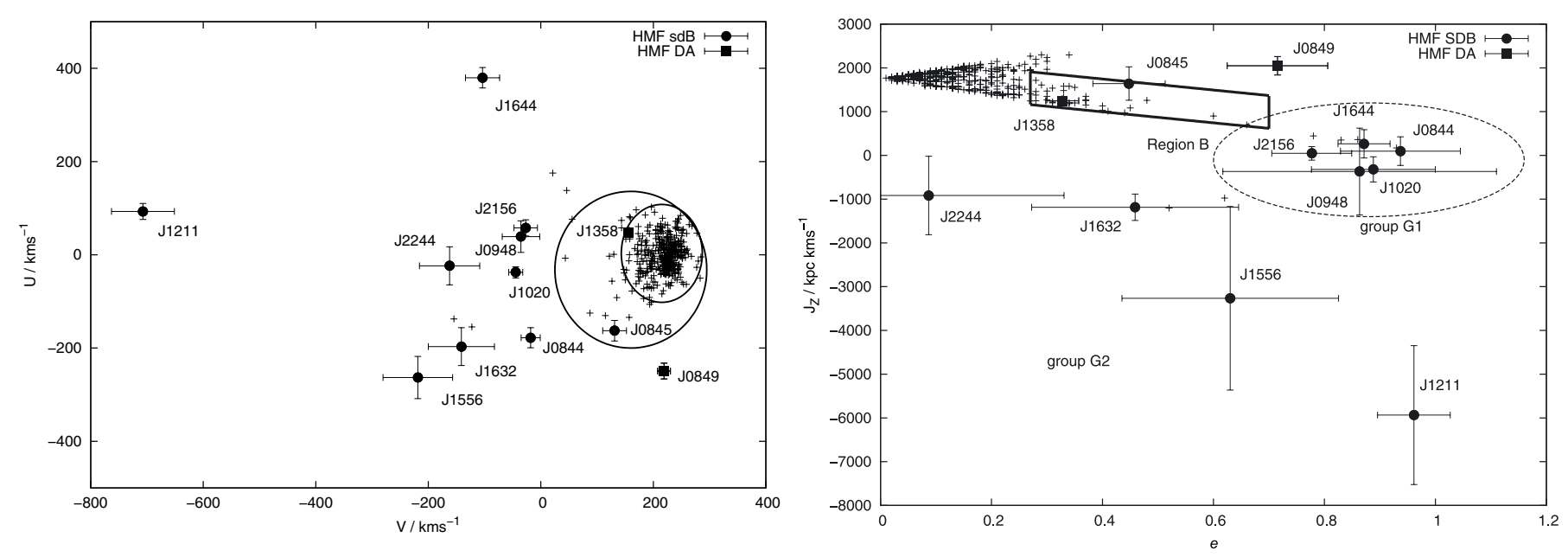

Fig. 4. $V-U$ (left) and $e-J_{Z}$ diagram (right) for our 12 targets. The white dwarf sample (+) of Pauli et al. (2006) serves as reference. The solid ellipses render the $3 \sigma$-thin and thick disc contours, while the solid box marks the thick disc region (Region B). Ten of our targets are outside of the regions for the two disc populations in both diagrams, but form two distinct groups. The five stars inside the dashed ellipse are called group 1 (G1), while the stars with negative $J_{Z}$ are defined as group 2 (G2).

However, for 10 stars at least two of three indications are present, which renders them as halo stars, following Pauli et al. (2006). In the $e-J_{Z}$ diagram the cluster of subdwarfs with high eccentricities at $J_{Z} \approx 0$ clearly catches one's eye. We call this cluster "group 1" (G1), because the respective stars share similar kinematic properties. They have nothing to do with the disc rotation and show only marginal velocity components in the direction of the Galactic plane. Such behaviour is very typical of halo objects because they travel mainly perpendicular through the disc. Four subdwarfs have very negative angular momenta, which means that they are on retrograde orbits. We term this group of subdwarfs "group 2" (G2).

The two targets with the highest measured PMs turned out to be DA white dwarfs. In fact, J1358+4729 has already been discovered and analysed by Eisenstein et al. (2006). They derived atmospheric parameters $\left(T_{\text {eff }}=10635 \pm 61 \mathrm{~K}\right.$, $\log g=8.16 \pm 0.053)$, which are perfectly consistent with our values. J1358+4729 shows thick disc kinematics (see Fig. 4) according to both diagrams and does not belong to the halo population. The other white dwarf J0849+1455 has not been analysed before, except for a proper motion study by Evans (1992), and it belongs to the halo population (see Fig. 4). In their last orbits their trajectories never came close to the central part of the Galaxy.

\subsection{J1211+1437: extreme halo or hyper-velocity star}

According to the kinematic analysis presented above, the sdB star J1211+1437 shows the most extreme kinematics in G2 of retrograde orbit stars, because it lies far away from the other stars in the kinematic diagrams.

\section{$\mathrm{J} 1211+1437$ - an HVS candidate}

The Galactic rest-frame velocity of $\mathrm{J} 1211+1437$ is so high $\left(v_{\mathrm{GRF}}=713_{-139}^{+155} \mathrm{~km} \mathrm{~s}^{-1}\right.$, see Fig 8$)$ that it exceeds the local Galactic escape velocity of $v_{\text {esc }}=507 \mathrm{~km} \mathrm{~s}^{-1}$ if we adopt the Galactic potential of Allen \& Santillan (1991). J1211+1437 is an HVS candidate that could have been ejected from the GC by the Hills mechanism. To test this hypothesis we traced the trajectory back to zero Galactic latitude.
As can be seen from Fig. $7 \mathrm{~J} 1211+1437$ does not originate in the GC. Its place of origin more likely lies in the Galactic disc, where no SMBH is known to exist. As a result, the Hills (1988) slingshot mechanism can be excluded. The ejection velocity for that star, if it would have belonged to a co-rotating disc population, is about $v_{\mathrm{ej}} \approx 550 \mathrm{~km} \mathrm{~s}^{-1}$, so the star might have been ejected from the Galactic disc as a runaway star.

\section{$\mathrm{J} 1211+1437$ and the dark matter halo}

If, however, $\mathrm{J} 1211+1437$ is an old halo star that has experienced several disc passages, the star has to be bound to the Galaxy. This can only be achieved if the dark matter halo is more massive than the adopted one in the potential of Allen \& Santillan (1991). In general the kinematics of the most extreme stars provide the best constraints on the mass of the dark halo (Wilkinson \& Evans 1999). Przybilla et al. (2010) find a population II BHB star travelling towards us at such a high speed that a dark matter halo mass of at least $1.7 \times 10^{12} M_{\odot}$ is required to keep the star bound to the Galaxy. This value is considerably higher than adopted by Allen \& Santillan (1991). Therefore numerical experiments were carried out in which the Galactic potential was modified by increasing the mass of the dark matter halo. This constrains the total Galactic mass to exceed $M_{\text {total }}^{\text {new }}=2.0 \pm_{-1.2}^{+2.4} \times 10^{12} M_{\odot}$. The errors are based on the Monte Carlo distribution of the space velocity. Although the uncertainties are large, our value is perfectly consistent with other proper motion based kinematic mass estimates (Przybilla et al. 2010; Sakamoto et al. 2003; Wilkinson \& Evans 1999), which also support a high-mass Galactic halo. However, it has to be stressed that this conclusion can only be drawn if the orbit of $\mathrm{J} 1211+1437$ is bound ${ }^{5}$.

\subsection{J1644+4523 - the fastest G1 object}

Another star that is worth closer inspection is the sdB star $\mathrm{J} 1644+4523$, which belongs kinematically to the G1 group. The star is heading towards us and therefore must be bound. It is

\footnotetext{
5 The rest of the sample is bound to the Galaxy, although the subdwarfs J1556+4708 and J1644+4523 might exceed the Galactic escape velocity if their respective errors (see Table 4) are taken into account and the Galactic potential of Allen \& Santillan (1991) is applied.
} 
A. Tillich et al.: The Hyper-MUCHFUSS project: probing the Galactic halo with sdB stars
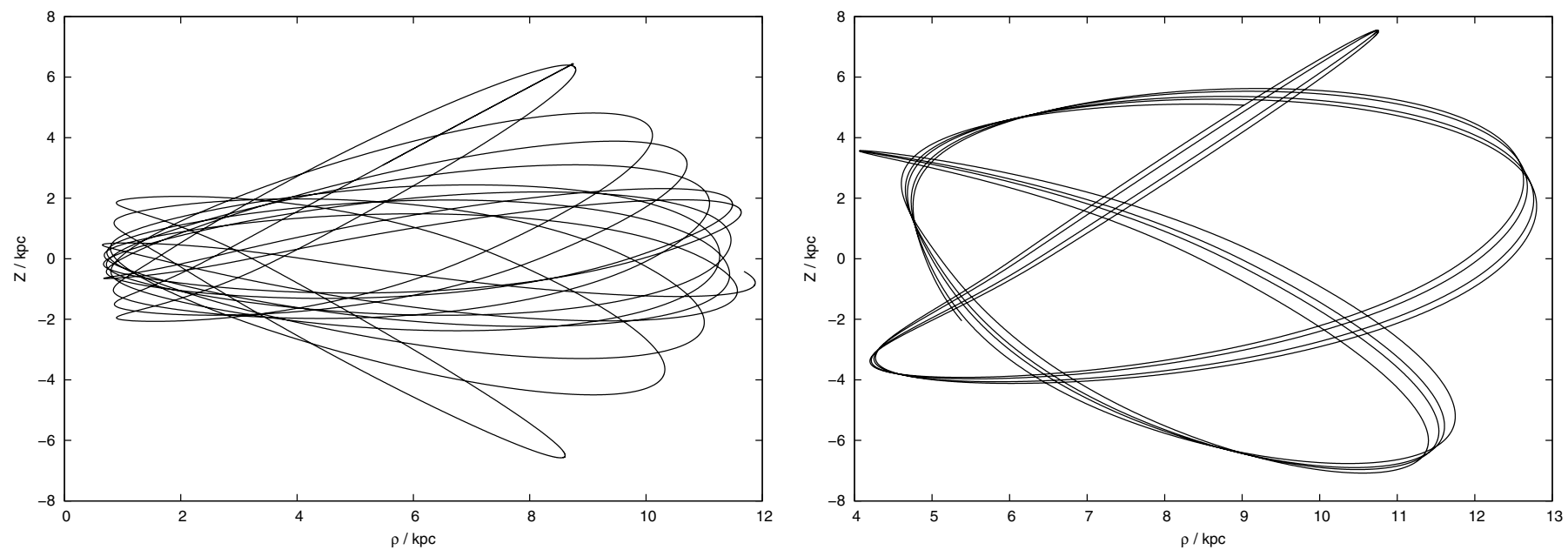

Fig. 5. $\rho$-Z-diagrams for the two group representatives, J1020+0137 (group 1, left) and J1632+2051 (group 2, right).
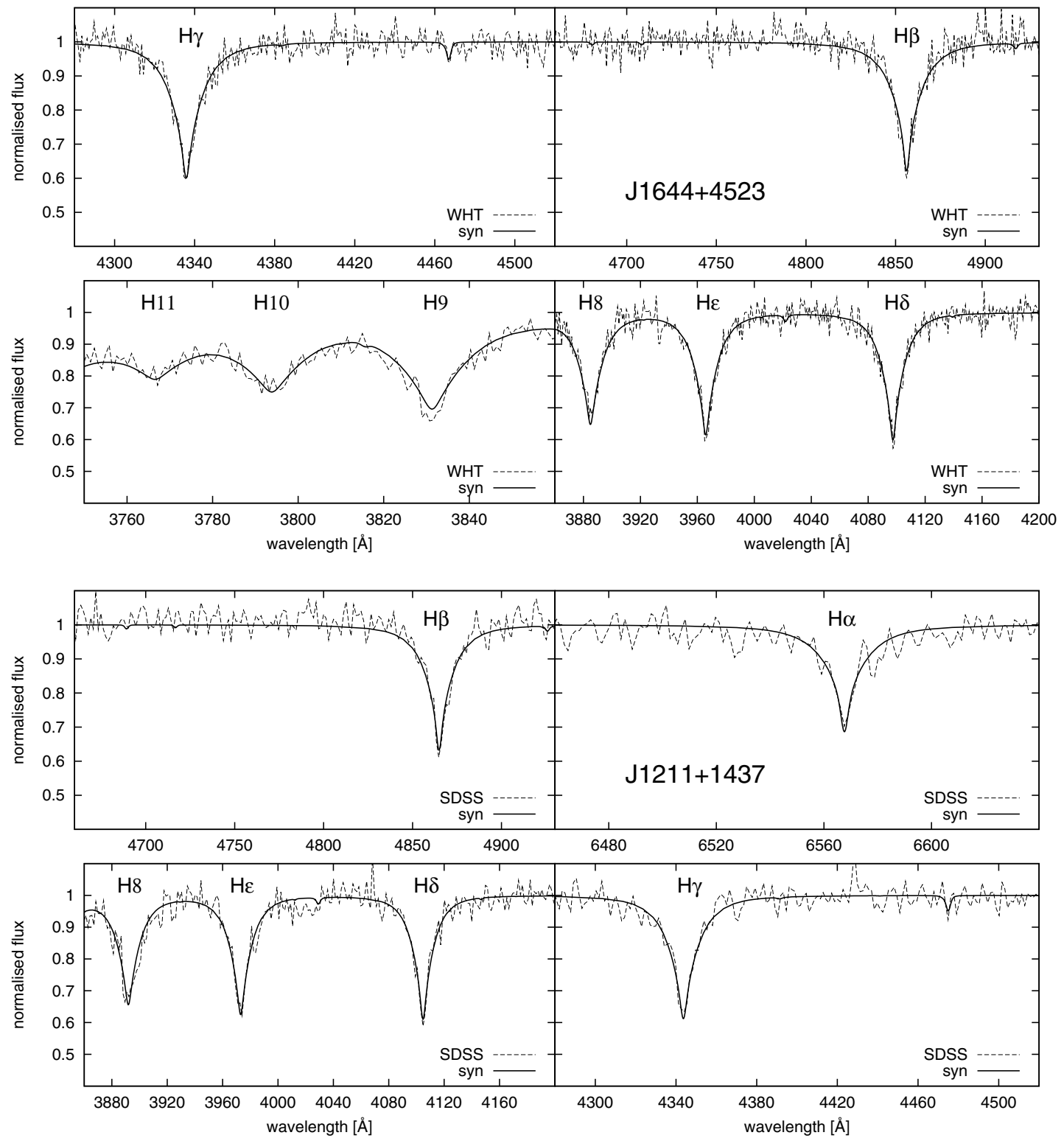

Fig. 6. Comparison between the synthetic model with the observed spectrum (WHT/SDSS) for the sdBs J1644+4523 (top) and J1211+1437 (bottom). The overall agreement is good. 


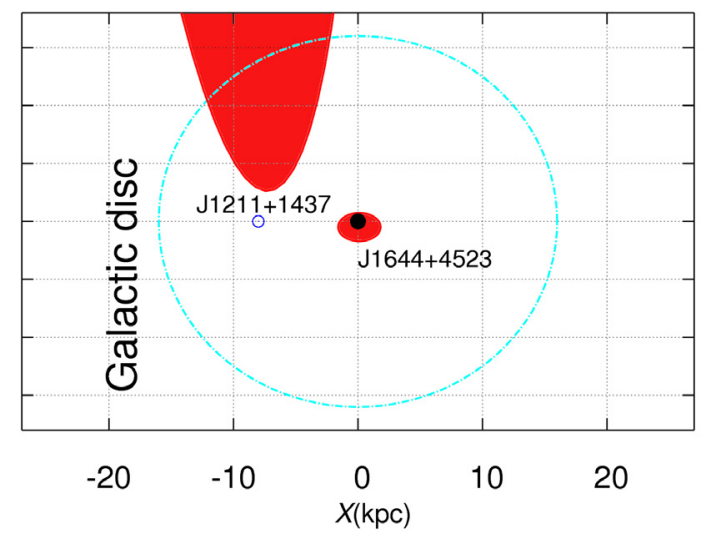

10

5 $0 \frac{0}{\frac{0}{2}}$ $-5$ $-10$

Fig. 7. Intersection region of the subdwarfs' past trajectories for $\mathrm{J} 1211+1437$ and J1644+4523 with the GD in order to decide on their origin (grey region). For orientation, the position of the sun is marked as well.

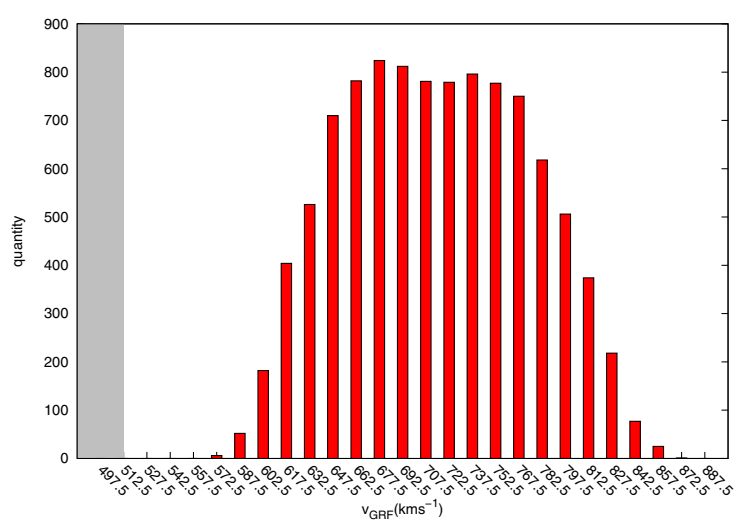

Fig. 8. Galactic rest-frame velocity distribution for J1211+1437 derived from a Monte Carlo procedure with a depth of 10000 (cp. Tillich et al. 2009, 2010b; Przybilla et al. 2010). The grey shaded area indicate the velocities for which the star is bound in the potential of Allen \& Santillan (1991).

the second fastest hot subdwarf of the halo population. We measured a GRF velocity of $v_{\mathrm{GRF}}=468_{-90}^{+104} \mathrm{~km} \mathrm{~s}^{-1}$, which is below the escape velocity of $v_{\mathrm{esc}}=534 \mathrm{~km} \mathrm{~s}^{-1}$ in the Galactic potential of Allen \& Santillan (1991). As for J1211+2423, we might alternatively consider that the star was ejected as a runaway star. We calculated the trajectory backwards into the past and found that the star might have originated in the Galactic bulge (GB), which can be seen from Fig. 9. The last pericentre passage occurred at a distance of only $\sim 0.5 \pm 1.0 \mathrm{kpc}$ from the GC (see Fig. 7) and the apocentre distance of the star's trajectory is located far out in the halo at $\sim 115 \mathrm{kpc}$. The high ejection velocity of $v_{\mathrm{ej}}=597 \mathrm{~km} \mathrm{~s}^{-1}$ could possibly be consistent with the ejection by the SMBH in the GC, as the error ellipse encloses the GC (see Fig. 7). The time-of-flight (TOF) is much longer $(\mathrm{TOF}=1.27 \mathrm{Gyr})$ than the helium core-burning phase lasts $(\approx 200 \mathrm{Myr})$. This means that $\mathrm{J} 1644+4523$ evolved to a subdwarf a long time after it was possibly ejected from the GB. This is very problematic, as EHB stars most likely require a binary interaction scenario to form (Han et al. 2003). In this case the ejection by the SMBH would disrupt any initial binary and the sdB cannot form. One solution could be a shorter TOF. The kinematic analysis of J1211+2423 has indicated that the dark matter halo may be more massive than assumed in the Galactic potential of Allen \& Santillan (1991).
Therefore we repeated the kinematical calculations for a higher dark halo mass and adopted $M_{\text {halo }}^{\text {new }}=3.4 \times 10^{12} M_{\odot}$ as suggested by Abadi et al. (2009) with a mass distribution out to $100 \mathrm{kpc}$, following Allen \& Santillan (1991). The place of the last disc intersection remained almost unchanged, but the time-of-flight is strongly reduced to $\mathrm{TOF}=145 \mathrm{Myr}$, which is consistent with the lifetime of hot subdwarfs; hence, the star could have been ejected as subdwarf in a high-mass halo and did not have to evolve after the ejection. In that case the last pericentre passage occurred at a slightly greater distance of $\sim 0.65 \mathrm{kpc}$ from the GC, and the apocentre distance of the stars trajectory is located in the halo at only $\sim 22 \mathrm{kpc}$.

\subsection{The entire $s d B$ sample}

We consider it worthwhile to inspect the runaway scenario for all target sdB stars by taking a closer look at their trajectories from the Galactic plane to their present location in the Galactic halo. Figure 11 displays the areas in the plane from which the stars could have originated for the kinematic group G1 (top) and G2 (bottom). As can be seen the G1 stars would originate from the inner Galactic disc or bulge, in particular J1644+4523, whereas the G2 stars (except J1632+2051) would come from the outer $\operatorname{disc}^{6}$. The case of J1211+1437 demonstrates that some of our targets could possibly be ejected runaway stars. If so the places of plane crossings of the G1 stars would not rule out an origin in the GC (except for J0120+0137). However, a GC origin of the G2 stars is excluded as their potential places of origin do not include the central part of the Galaxy.

\subsection{Kinematics of hot subdwarfs}

Up to now, the only 3D kinematical study of such sdBs in the field has been performed by Altmann et al. (2004). From a sample of $114 \mathrm{sdBs}$, they found 15 with orbits, which differ considerably from disc orbits, while the vast majority are consistent with the disc population. Altmann et al. (2004) noticed that their sample may contain close binary stars that are RV variable, because the fraction is known to be about $40 \%$. At the time of writing, several stars of their sample had not been checked for RV variability, but have been shown to be RV variable later on. We did not correct for this effect.

We compare the kinematical properties in the $V-U$-plane of their sample to ours in Fig. 10. If applying the criteria of Pauli et al. (2006), the vast majority of the Altmann et al. (2004) sample belong to the thin disc, with about two dozen thick disc and a dozen halo stars. The most eye-catching difference between the two samples in Fig. 10 is the absence of stars with rapid retrograde orbits, i.e. our class G2. Only very few halo sdBs in the sample of Altmann et al. (2004) would be classified as G1 (showing essentially no Galactic rotation). However, our sample lacks stars with high positive $V$ velocities.

When comparing the two samples, we have to consider that the stars in the sample of Altmann et al. (2004) are much brighter, hence, nearer than those in our sample. Consequently, we probe a much larger volume of space.

\section{Summary and conclusion}

Thirty-nine high-velocity, faint blue stars have been identified from the MUCHFUSS survey. We were able to measure proper

\footnotetext{
${ }^{6}$ The remaining thick disc subdwarf J0845+1352 shows a trajectory, which intersects within the Galactic disc.
} 


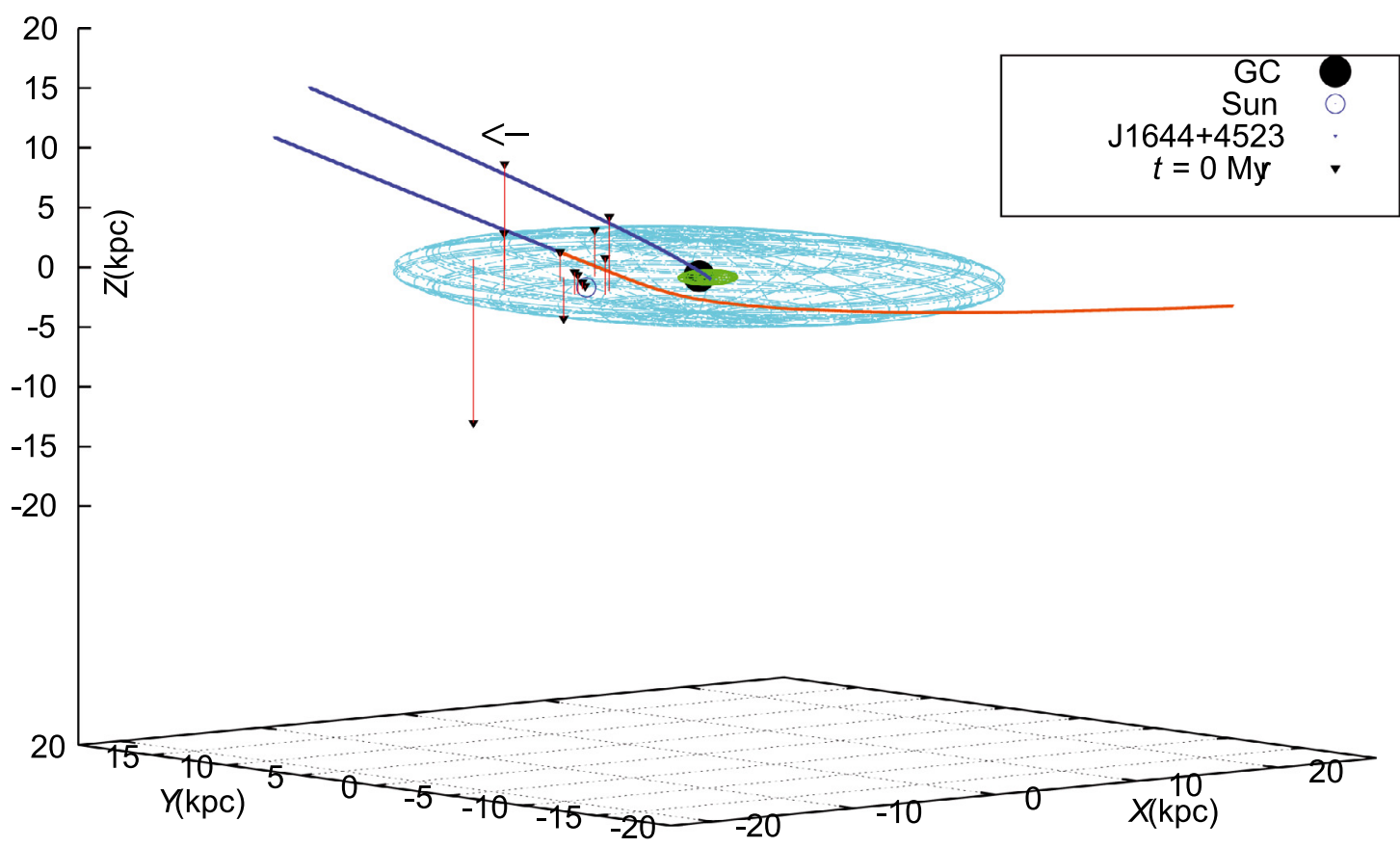

Fig. 9. 3D plot of the current positions of our 12 stars (black triangles) relative to the Galactic disc (grey), showing also the trajectory for $\mathrm{J} 1644+4523$ and the area of potential origin (dark grey).

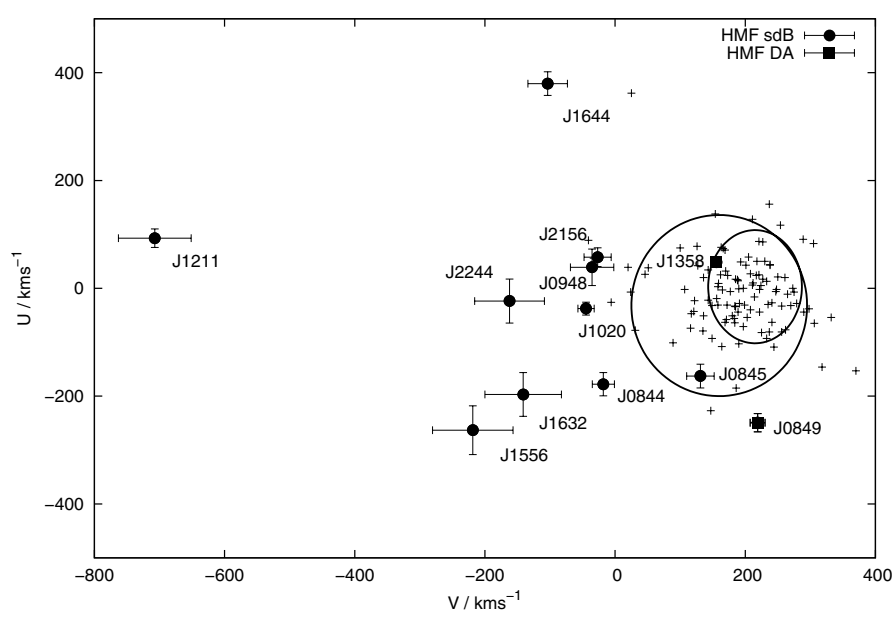

Fig. 10. Kinematic $V-U$ diagram for the programme sdB stars in comparison with the sample (+ signs) of Altmann et al. (2004). The solid ellipses render the $3 \sigma$-thin and thick disc contours of Pauli et al. (2006).

motions that are significantly different from zero for 12 of them by determining positions on a 60-year time base. Quantitative spectral analyses were performed to derive their distances. From an investigation of their kinematics nine sdB stars were identified as halo members and one as belonging to the thick-disc. The two remaining stars turned out to be white dwarfs in the halo and the thick-disc. Two distinctive kinematic groups among the sdB stars emerged: the normal halo subdwarfs (G1) with low Galactic rotation and the extreme halo subdwarfs $(\mathrm{G} 2)$ on highly-eccentric retrograde orbits.

The extreme halo star $\mathrm{J} 1211+1437$ is of particular interest because it would be unbound to the Galaxy if the standard Galactic potential was applied, so it would be the second HVS among the hot subdwarfs and the first one with spectral type $\mathrm{sdB}$. However, assuming the star is a population II object bound to the Galaxy, we can derive a lower limit to the mass of the dark matter halo of $2 \times 10^{12} M_{\odot}$. Other stars that are approaching the Earth, J1644+4523 in particular, have to be bound to the Galaxy and therefore might provide constraints on the dark matter mass.

The existence of two kinematically distinct groups indicates different origins. In the ejection scenario, the class G1 stars would originate in the inner Galaxy, whereas the G2 ones would come from its outer parts. However, if some of the sdB stars in our sample were runaway stars, we have to find an ejection mechanism. The G1 stars (except J0120+0137) may originate in the GC and the SMBH slingshot mechanism might work for them. For the G2 stars, an origin in the GC can be excluded, meaning that we have to find another mechanism to explain their origin.

Kinematic studies among massive B-type stars indicate that variations of the typical runaway-scenario might provide an answer (Heber et al. 2008; Irrgang et al. 2010). Conveniently, sdBs most likely require a binary interaction scenario to form (Han et al. 2003). In principle, one can think of a variation in the RLOF mechanism as suggested by Nelemans \& Tauris (1998) in the context of the formation of undermassive white dwarfs. If the subdwarf's RGB progenitor is losing its envelope to a massive white dwarf, an asymmetric accretion induced collapse could occur. The system can then be disrupted, with the accretor leaving the system as a high-velocity neutron star. As computed by Marietta et al. (2000), the companions SN explosion does not have to happen at the tip of the RGB for the envelope to be lost and the core to end up as an $\mathrm{sdB}$ star. In their calculations, the SN explosion itself can strip 96 to $98 \%$ of the envelope from an RGB star, effectively leaving a naked He core. If the core is massive enough for helium burning it can experience a late core He flash, and end up as a single EHB star. As pointed out by Østensen (2009), the abandoned subdwarf would end up single and in an unusual galactic orbit in both cases.

Recently, Wang \& Han (2009) and Justham et al. (2009) have suggested a single degenerate SN Ia scenario to explain the formation of the helium sdO US 708. Accordingly, a white dwarf accretes matter from a helium star in a close binary system. After 

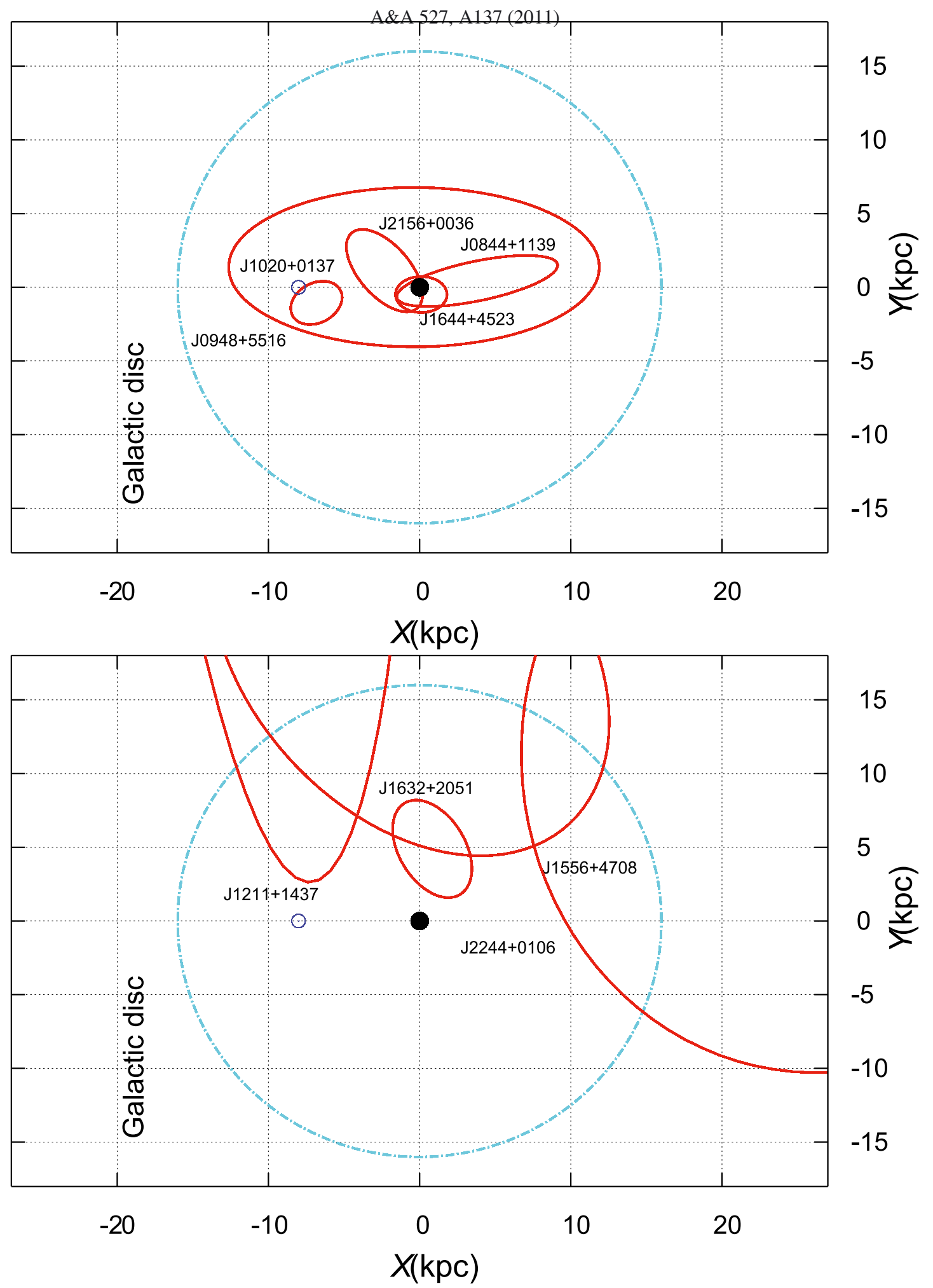

Fig. 11. Intersection region of the halo subdwarfs' past trajectories with the GD for conclusions on their origin. In group G1, 4 out of 5 subdwarfs are consistent with a GC origin (top). In the extreme group G2, we found 3 subdwarfs, which might originate in the outer Galactic rim (bottom), most likely produced by supernova runaway ejection. For orientation, the position of the sun is marked as well.

exceeding the Chandrasekhar mass limit, the white dwarf explodes as a type Ia supernova. The orbital period has shrunk to about $1 \mathrm{~h}$ and the corresponding orbital velocity of the hot HesdO companion may exceed $500 \mathrm{~km} \mathrm{~s}^{-1}$. After the explosion of the primary, the binary is disrupted and the sdO companion released at its orbital velocity. In addition it may be sped up by the SN kick. However, all our programme stars are sdB stars, which retain a hydrogen-rich envelope of $10^{-4}$ to $10^{-2} M_{\odot}$. It thus appears unlikely that the scenario applies to high-velocity sdB stars.

As some globular clusters are known to host sdB stars, ejection from such clusters may give rise to high-velocity sdB stars. Alternatively, the programme stars may stem from the disruption of a satellite galaxy in the Galactic halo.

We shall extend our study as the MUCHFUSS survey proceeds. As demonstrated, the high-velocity hot subdwarfs are 
important tools for constraining the mass of the Galactic dark matter halo. Its full potential will develop once the GAIA mission has provided much more accurate distances and proper motions.

Acknowledgements. A.T., S.G., and H.H. are supported by the Deutsche Forschungsgemeinschaft (DFG) through grants HE1356/45-1, HE1356/49-1, and HE1356/44-1, respectively. Travel to the DSAZ (Calar Alto, Spain) was supported by DFG under grants HE1356/48-1 and HE1356/50-1.

R.H.Ø. has received funding from the European Research Council under the European Community's Seventh Framework Programme (FP7/2007-2013)/ERC grant agreement No. 227224 (PROSPERITY), as well as from the Research Council of K.U. Leuven grant agreement GOA/2008/04. We thank Detlev Koester, who provided the models for the white dwarfs. R.-D.S. thanks Doug Finkbeiner for his help accessing the SDSS data at Princeton University. Funding for the SDSS and SDSS-II has been provided by the Alfred P. Sloan Foundation, the Participating Institutions, the National Science Foundation, the U.S. Department of Energy, the National Aeronautics and Space Administration, the Japanese Monbukagakusho, the Max Planck Society, and the Higher Education Funding Council for England. The SDSS Web Site is http://www.sdss.org/.

\section{References}

Abadi, M. G., Navarro, J. F., \& Steinmetz, M. 2009, ApJ, 691, L63 Allen, C., \& Santillan, A. 1991, Rev. Mex. Astron. Astrofis., 22, 255 Altmann, M., Edelmann, H., \& de Boer, K. S. 2004, A\&A, 414, 181 Battaglia, G., Helmi, A., Morrison, H., et al. 2005, MNRAS, 364, 433 Brown, T. M., Smith, E., Ferguson, H. C., et al. 2008, ApJ, 682, 319 Brown, W. R., Geller, M. J., Kenyon, S. J., \& Kurtz, M. J. 2005, ApJ, 622, L33 Brown, W. R., Geller, M. J., Kenyon, S. J., Kurtz, M. J., \& Bromley, B. C. 2007, ApJ, 660, 311

Brown, W. R., Geller, M. J., \& Kenyon, S. J. 2009, ApJ, 690, 1639

Carlsberg-Meridian-Catalog 2006, Copenhagen Univ. Obs., Inst. of Astr., Cambridge, UK, Real Inst. y Obs. de la Armada en San Fernando, 1304, 0

Cutri, R. M., Skrutskie, M. F., van Dyk, S., et al. 2003, 2MASS All Sky Catalog of point sources.

Dehnen, W., \& Binney, J. J. 1998, MNRAS, 298, 387

Dorman, B., Rood, R. T., \& O'Connell, R. W. 1993, ApJ, 419, 596

Edelmann, H., Napiwotzki, R., Heber, U., Christlieb, N., \& Reimers, D. 2005, ApJ, 634, L181

Eisenstein, D. J., Liebert, J., Harris, H. C., et al. 2006, ApJS, 167, 40

Evans, D. W. 1992, MNRAS, 255, 521

Finkbeiner, D. P., Padmanabhan, N., Schlegel, D. J., et al. 2004, AJ, 128, 2577

Geier, S., Hirsch, H., Tillich, A., et al. 2011a, A\&A, submitted
Geier, S., Maxted, P. F. L., Napiwotzki, R., et al. 2011b, A\&A, 526, A39

Green, R. F., Schmidt, M., \& Liebert, J. 1986, ApJS, 61, 305

Hambly, N. C., MacGillivray, H. T., Read, M. A., et al. 2001, MNRAS, 326, 1279

Han, Z., Podsiadlowski, P., Maxted, P. F. L., \& Marsh, T. R. 2003, MNRAS, 341, 669

Heber, U. 1986, A\&A, 155, 33

Heber, U. 2009, ARA\&A, 47, 211

Heber, U., Reid, I. N., \& Werner, K. 2000, A\&A, 363, 198

Heber, U., Edelmann, H., Napiwotzki, R., Altmann, M., \& Scholz, R.-D. 2008, A\&A, 483, L21

Hills, J. G. 1988, Nature, 331, 687

Hirsch, H. A., Heber, U., O’Toole, S. J., \& Bresolin, F. 2005, A\&A, 444, L61

Irrgang, A., Przybilla, N., Heber, U., Nieva, M. F., \& Schuh, S. 2010, ApJ, 711, 138

Jordi, K., Grebel, E. K., \& Ammon, K. 2006, A\&A, 460, 339

Justham, S., Wolf, C., Podsiadlowski, P., \& Han, Z. 2009, A\&A, 493, 1081

Koester, D. 2009, A\&A, 498, 517

Lawrence, A., Warren, S. J., Almaini, O., et al. 2007, MNRAS, 379, 1599

Lisker, T., Heber, U., Napiwotzki, R., et al. 2005, A\&A, 430, 223

Marietta, E., Burrows, A., \& Fryxell, B. 2000, ApJS, 128, 615

McMahon, R. G., Irwin, M. J., \& Maddox, S. J. 2000, VizieR Online Data Catalog, 1267, 0

Napiwotzki, R. 2008, in Hydrogen-Deficient Stars, ed. A. Werner, \& T. Rauch, ASP Conf. Ser., 391, 257

Navarro, J. F., Frenk, C. S., \& White, S. D. M. 1996, ApJ, 462, 563

Navarro, J. F., Frenk, C. S., \& White, S. D. M. 1997, ApJ, 490, 493

Nelemans, G., \& Tauris, T. M. 1998, A\&A, 335, L85

Odenkirchen, M., \& Brosche, P. 1992, Astron. Nachr., 313, 69

Østensen, R. H. 2009, Communications in Asteroseismology, 159, 75

Pauli, E., Napiwotzki, R., Altmann, M., et al. 2003, A\&A, 400, 877

Pauli, E., Napiwotzki, R., Heber, U., Altmann, M., \& Odenkirchen, M. 2006, A\&A, 447, 173

Przybilla, N., Tillich, A., Heber, U., \& Scholz, R. 2010, ApJ, 718, 37

Ramspeck, M., Heber, U., \& Moehler, S. 2001, A\&A, 378, 907

Roeser, S., Demleitner, M., \& Schilbach, E. 2010, AJ, 139, 2440

Sakamoto, T., Chiba, M., \& Beers, T. C. 2003, A\&A, 397, 899

Smith, M. C., Ruchti, G. R., Helmi, A., et al. 2007, MNRAS, 379, 755

Steinmetz, M., Zwitter, T., Siebert, A., et al. 2006, AJ, 132, 1645

Tillich, A., Przybilla, N., Scholz, R., \& Heber, U. 2009, A\&A, 507, L37

Tillich, A., Geier, S., Heber, U., et al. 2010a, Ap\&SS, 117

Tillich, A., Przybilla, N., Scholz, R., \& Heber, U. 2010b, A\&A, 517, A36

Wang, B., \& Han, Z. 2009, A\&A, 508, L27

Wilkinson, M. I., \& Evans, N. W. 1999, MNRAS, 310, 645

Xue, X. X., Rix, H. W., Zhao, G., et al. 2008, ApJ, 684, 1143

York, D. G., Adelman, J., Anderson, Jr., J. E., et al. 2000, AJ, 120, 1579 